\title{
Modeling and Simulation of Wheel Driving Systems based on Terramechanics for Planetary Explanation Rover using Modelica
}

\author{
Hiroki Yoshikawa $^{1}$ Takatsugu Oda $^{1}$ Kenichiro Nonaka ${ }^{1} \quad$ Kazuma Sekiguchi $^{1}$ \\ ${ }^{1}$ Mechanical Systems Engineering, Tokyo City University, Japan, \{g1681237, g1591201, knonaka, \\ ksekiguc\} etcu.ac.jp
}

\begin{abstract}
Planetary exploration rovers have to accomplish various missions on uneven and loose terrain. In recent years, systems of rovers adopting terramechanics which determine the force and moment characteristics of the wheel on loose soil is studied. In this study, using Modelica language, we construct a wheel model based on terramechanics, and we identify the wheel characteristics as a linear for a control. We conduct a numerical simulation of the rover using a controller including the identified longitudinal force model. It is shown that when the rover follows a straight line on a plane, the longitudinal force model identified using known soil parameters has sufficient accuracy on the wheel response based on terramechanics and could be used as a control model. Keywords: terramechanics, modeling, identification, space robots, control system
\end{abstract}

\section{Introduction}

In recent years, research and development of planetary exploration rovers in various configurations have been carried out to investigate the planets. Planetary exploration rovers have to achieve a stable traveling on uncertain and severe terrain. The planet surface is covered with fine deposits, called regolith, and uneven terrain such as craters and rocks. Various planetary exploration rovers have been developed which is equipped with, for example, wheel mechanisms with suspensions to adapt to the planetary surface, crawler mechanisms to enhance the drawbar pull or leg mechanisms to climb over steps (Seeni et al., 2008). Also, NASA is planning to operate a hybrid rover "ATHLETE" which is equipped with wheel and leg mechanisms.

When rovers move on planetary surface, it is important to take into account of terramechanics which governs a relation between soft soil and the driving system of rovers. In order to analyze the effect of the soil, a semiempirical model proposed by Bekker using the experimental results and a model using Discrete Element Method (DEM) without dependence on wheel parameters are studied (Nakashima et al., 2010). Combining DEM with Finite Element Method (FEM), the simulation using Soil Contact Model (SCM) of Multi-Body System (MBS) which analyzes the more detailed soil movement is proposed (Krenn and Gibbesch, 2011). The deformation of soil and the op- timal wheel shape are analyzed through these simulations to consider efficient travel on loose soil. However, it is not suitable for the motion analysis of the rover, since it takes large calculation time with FEM and DEM which handle huge complicated elements in order to ensure reasonable accuracy (Taheri et al., 2015).

As for the studies about the control based on terramechanics, designing path (Ding et al., 2014) and analysis of traveling performance while ascending (Ishigami et al., 2007 ) is conducted. A slip ratio control of the wheels on loose soil using sliding mode control for the rover model considering terramecahnics is proposed (Gu et al., 2007). In addition, another slip ratio control of the wheels using PID control to adapt the parameters of terrain surface is studied (Iagnemma and Dubowsky, 2004).

While it is desirable to conduct experiments in space environments to verify these models, computer simulations are preferred considering huge cost. However, it is difficult to compensate for the differences of planetary environments like gravitational field and so on (Pulecchi and Lovera, 2006). To conduct a simulation with minimized the error between the simulation model and the actual equipment is minimized, it is extremely effective for comprehensive analysis through the more detailed rover model and contact model of loose soil. The simulations using Modelica language and modeling tool of physical domains attract a lot of attention. We do not need to care about causality to create the wheel model based on terramechanics such as slip ratio, sideslip angle and velocity of wheel, since Modelica is an equation based language. These features enable us to combine the wheel and rover model effectively.

In previous our study, we conduct simulations considering the space environment using the fundamental control system and the robot model designed by Modelica. In this study, using Modelica language we design a rover model equipped with the terramechanics model to conduct a simulation with more detail model. We identify the identified model which expresses the relationship between input torque and longitudinal force based on the simulation results of the terramechanics model. Becasue the terramechanics model is too complex to use in a controller, we design the motion controller using the identified model. We evaluate the effectiveness of identified model through numerical simulations. Therefore, 


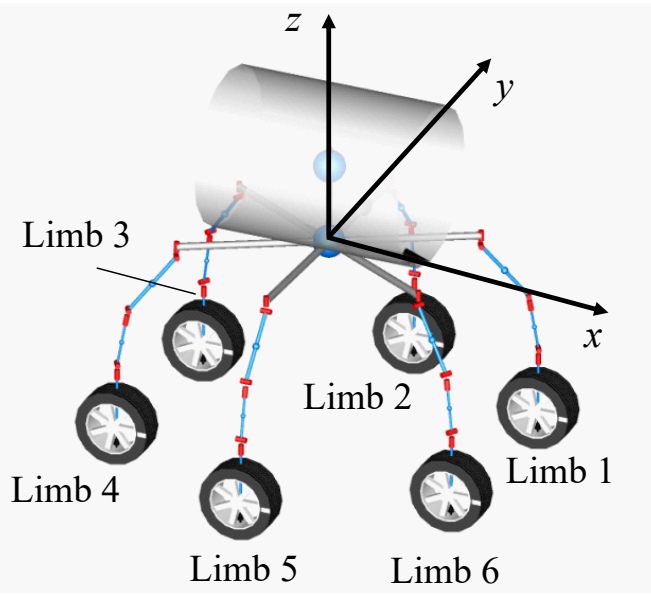

Figure 1. Leg-wheel mobile robot model with six joints of each limb.

the identified model approximates the characteristics of the terramechanics model.

\section{Modeling controlled object}

\subsection{Leg-wheel mobile robot model}

Figure 1 depicts a rover model of the controlled object (Yoshikawa et al., 2016). We use a lunar exploration rover "ATHLETE" developed by NASA/JPL as a reference model (Wilcox et al., 2007). This rover is equipped with six limbs with six joints while wheels achieve a high movement performance and accommodate a wide range of tasks using the redundancy. We create this rover model by using Modelica language to control the degree of freedom of the leg-wheel mechanisms with similar movements of ATHLETE. The coordinate system of the rover is attached at the center of the body. The limb has a number to be distinguished from the others in this coordinate system, as depicted in Figure 1.

\subsection{Wheel model based on terramechanics}

\subsubsection{Assumptions of the wheel model}

We introduce terramechanics to the wheel model of the controlled objects. We make reference to semi-empirical model (Ishigami et al., 2007) (Wong, 2001) to the wheel model based on terramechanics. Figure 2 depicts the rigid wheel rolling on loose soil. The assumptions of the wheel model are as follows:

- The contact surface between wheels and the ground is flat.

- Radius $r$ and width $b$ of wheel have enough rigidity.

- Wheel rotation does not affect a frontal soil.

- The frontal soil is constricted and released at the rear of the wheel.

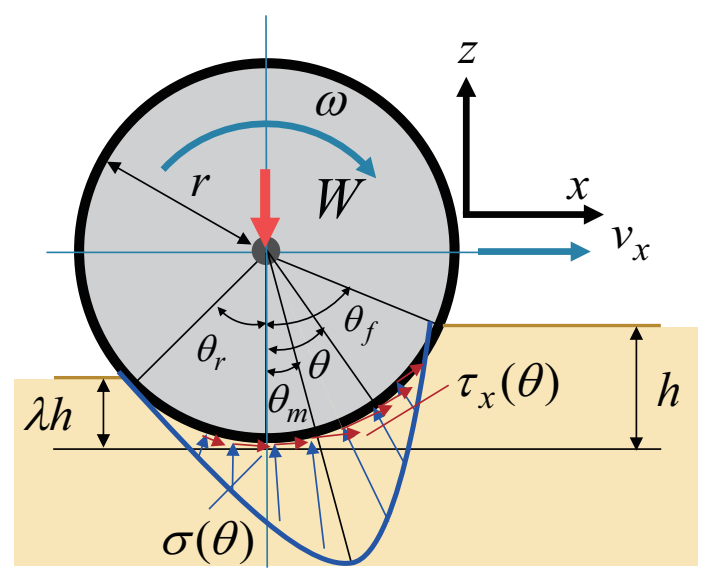

Figure 2. Normal stress and shear stress distribution concept of terramechanics while rolling (Ishigami et al., 2007).

- Lateral and vertical dynamics of wheels are not considered.

Figure 2 depicts the geometry of the wheel model based on these assumptions; the empirical equation is described in the following section.

\subsubsection{Entry and exit angle of wheel}

The forces generated from the wheel are calculated by integrating a stress distribution developed between the wheel and terrain surface. Entry angle $\theta_{\mathrm{f}}$ and exit angle $\theta_{\mathrm{r}}$ are introduced in order to decide the dynamic contact area of the wheel. The entry angle and exit angle are defined as follows:

$$
\begin{aligned}
& \theta_{\mathrm{f}}=\cos ^{-1}\left(1-\frac{h}{r}\right), \\
& \theta_{\mathrm{r}}=\cos ^{-1}\left(1-\frac{\lambda h}{r}\right),
\end{aligned}
$$

where $h$ is the sinkage of wheel and $\lambda$ is the volume ratio of soil.

\subsubsection{Specific wheel angle $\theta_{\mathrm{m}}$}

The normal stress distribution $\sigma$ (the blue curve in Figure 2) arises in the normal direction of the wheel while rolling. This normal stress distribution is approximated by the parabolic curve. The maximum stress angle $\theta_{\mathrm{m}}$ is an angle at which the value of normal stress is maximum as follows:

$$
\theta_{\mathrm{m}}=\left(a_{0}+a_{1} \kappa\right) \theta_{\mathrm{f}},
$$

where $a_{0}, a_{1}$ is a constant value and $\kappa$ is slip ratio. Slip ratio is represented by using a translational velocity of the wheel $v_{x}$ and angular velocity of the wheel $\omega$ :

$$
\kappa= \begin{cases}\left(\frac{r \omega-v_{x}}{r \omega}\right) & \left(r \omega>v_{x}\right) \\ \left(\frac{r \omega-v_{x}}{v_{x}}\right) & \left(r \omega<v_{x}\right) .\end{cases}
$$




\subsubsection{Normal stress distribution model based on bekker's equation}

The normal stress distribution model $\sigma(\theta)$ based on soil pressure equation proposed by Bekker is divided into two areas: the front parts of the specific wheel angle $\sigma_{\mathrm{f}}(\theta)$ $\left(\theta_{\mathrm{m}} \leq \theta<\theta_{\mathrm{f}}\right)$ and the rear parts $\sigma_{\mathrm{r}}(\theta)\left(\theta_{\mathrm{r}}<\theta \leq \theta_{\mathrm{m}}\right)$. The normal stress distribution model of the wheel is defined as follows:

$$
\begin{aligned}
\sigma_{\mathrm{f}}(\theta) & =r^{n}\left(\frac{k_{c}}{b}+k_{\phi}\right)\left[\left(\cos \theta-\cos \theta_{\mathrm{f}}\right)\right]^{n}, \\
\sigma_{\mathrm{r}}(\theta) & =r^{n}\left(\frac{k_{c}}{b}+k_{\phi}\right) \\
& {\left[\cos \left\{\theta_{\mathrm{f}}-\frac{\theta-\theta_{\mathrm{r}}}{\theta_{\mathrm{m}}-\theta_{\mathrm{r}}}\left(\theta_{\mathrm{f}}-\theta_{\mathrm{m}}\right)\right\}-\cos \theta_{\mathrm{f}}\right]^{n}, }
\end{aligned}
$$

where $k_{c}$ is pressure-sinkage module depending on the viscosity, $k_{\phi}$ is pressure-sinkage module depending on the friction and $n$ is the sinkage exponent depending on sinkage of soil.

\subsubsection{Shear stress model of wheel}

Shear stress model is defined as follows:

$$
\begin{aligned}
& \tau=\tau_{\max }\left(1-e^{-j / k}\right), \\
& \tau_{\max }=c+\sigma \tan \phi,
\end{aligned}
$$

where $c$ is the cohesion stress of the soil, $\phi$ is the internal friction angle of the soil, $j$ is the soil deformation and $k$ is the shear deformation modules. The shear stress of $x$ direction $\tau_{x}$ is obtained by assigning $\sigma$ to Eq. (8):

$$
\tau_{x}=(c+\sigma(\theta) \tan \phi)\left(1-e^{-j_{x}(\theta) / k_{x}}\right),
$$

where $k_{x}$ is the shear deformation modules of $x$ direction and $j_{x}$ is the soil deformation of $x$ direction as follows:

$$
j_{x}(\theta)=r\left[\theta_{\mathrm{f}}-\theta-(1-\kappa)\left(\sin \theta_{\mathrm{f}}-\sin \theta\right)\right] .
$$

\subsubsection{Vertical and longitudinal force of wheel}

The vertical force $F_{z}$ which is equal to the load of the wheel is calculated by the summation of the normal and shear stress of $z$ direction as follows:

$$
F_{z}=r b \int_{\theta_{\mathrm{r}}}^{\theta_{\mathrm{f}}}\left\{\tau_{x}(\theta) \sin \theta+\sigma(\theta) \cos \theta\right\} \mathrm{d} \theta .
$$

The normal and shear stress of the wheel can be calculated using the each contact angle $\theta_{\mathrm{f}}$ and $\theta_{\mathrm{r}}$ determined by the sinkage of the wheel $h$. Then, the longitudinal force is calculated by the summation of normal and shear stress of $x$ direction as follows:

$$
F_{x}=r b \int_{\theta_{\mathrm{r}}}^{\theta_{\mathrm{f}}}\left\{\tau_{x}(\theta) \cos \theta-\sigma(\theta) \sin \theta\right\} \mathrm{d} \theta .
$$

The rolling resistance torque $T_{x}$ is calculated using the shear stress as follows:

$$
T_{x}=r^{2} b \int_{\theta_{\mathrm{r}}}^{\theta_{\mathrm{f}}} \tau_{x}(\theta) \mathrm{d} \theta
$$

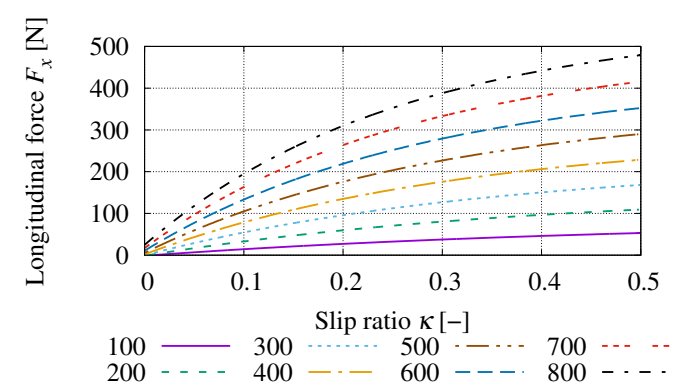

Figure 3. Relationship between slipratio and longitudinal force with respect to load of the wheel.

\subsubsection{Longitudinal characteristics}

Figure 3 shows longitudinal force $F_{x}$ with respect to the slip ratio of the wheel when the load of it increases every force $100 \mathrm{~N}$ within $100 \mathrm{~N} \sim 800 \mathrm{~N}$. As the slip ratio increases, the longitudinal force is saturated as Figure 3 indicates. In addition, as indicated in Figure 3, for the same slip ratio, the longitudinal force generated by the wheel depends on the load. It indicates that the increasing ratio of $F_{x}$ decreases as the load grows.

\section{Identification of the wheel model}

\subsection{Identified model}

In this section, to design a rover controller in which the identified model is additionally used, we identify the longitudinal force of the terramechanics model. We approximate the longitudinal force generated at the wheel by a linear first-order system. A step wheel torque is imposed on the wheel, then the wheel response data on the longitudinal force and the slip ratio is sampled. The identified longitudinal force model is depicted in Figure 4. The identified model is separated into two blocks: one for calculating the slip ratio by the wheel torque and the other for calculating the longitudinal force by the slip ratio. This separation helps to capture the feature of the physical relationship.

In the wheel model based on terramechanics, the wheel sinkage which depends on load is decided by the optimization. In order to identify the longitudinal force corresponding to the load change, we represent the parameters of the first order system using a look up table (LUT). Using the LUT in the identified model, we can consider the generated force due to influences of soil deformation caused by load change. Firstly, the gain $K_{\mathrm{LUT}}$ and the time constant $T_{\text {LUT }}$ are decided using the LUT. The reference values of the LUT are the wheel load $W$ and wheel torque $T_{\mathrm{w}}$. Secondly, the relationship between slip ratio $\kappa$ and longitudinal force $F_{x}$ with respect to load change is depicted in Figure 5. Each point in this Figure represents the reference results of the terramechanics model. To express these relationships in an equation, we approximate it as follows:

$$
F_{x}(W, \kappa)=a(W) \kappa+b(W)
$$




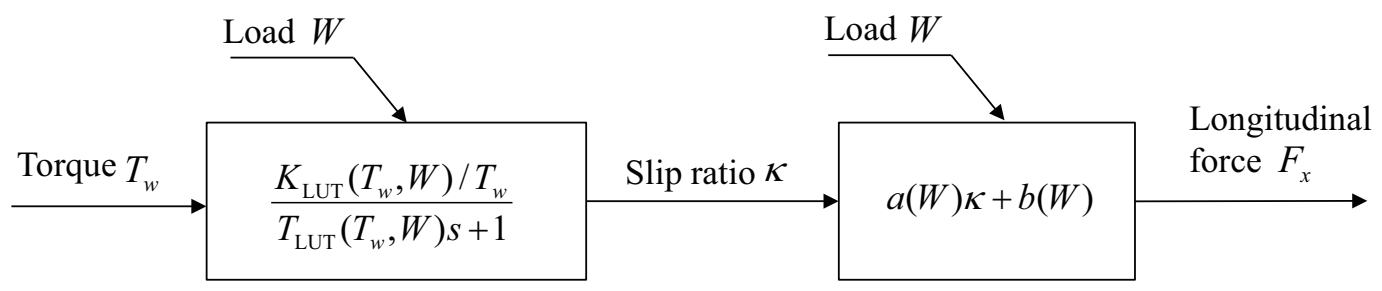

Figure 4. Identification model from wheel torque to longitudinal force of wheel.

Table 1. Precision of identified model to responce of wheel baced on terramechanics in the load 275, 575, 875 N.

\begin{tabular}{llll}
\hline Load & $275 \mathrm{~N}$ & $575 \mathrm{~N}$ & $875 \mathrm{~N}$ \\
\hline Precision & $82.1 \%$ & $96.8 \%$ & $96.2 \%$ \\
\hline
\end{tabular}

where $a(W)$ and $b(W)$ are the coefficient derived from the quadratic expressions with respect to load change, as shown in Figure 6 and Figure 7, respectively.

\subsection{Verification of identification model}

It is noted that the idetified model using LUT is an approximation which essentially includes interpolation error. Figure 8 indicates the longitudinal force obtained by the wheel based on terramechanics and the identified model of it in the load $W=575 \mathrm{~N}$ which is the interporated region. A precision of identified model is calculated using the following equation:

$$
\text { Fit }=\left(1-\frac{\sqrt{\sum_{k=1}^{N}[\hat{y}(k)-y(k)]^{2}}}{\sqrt{\sum_{k=1}^{N}[y(k)-\bar{y}]^{2}}}\right) \times 100,
$$

where $\hat{y}(k)$ is the output of identified model, $y(k)$ is the output obtained by the controlled object, $\bar{y}(k)$ is the average of it and $N$ is the number of data. In the case that the load is not the reference results of the teramechanics model, for example $W=275,575,875 \mathrm{~N}$, the precision for the step response of the wheel torque is shown in Table 1. As a result, all of the precision is over $82 \%$. If you need to increase the precision, the degree of the approximate expression will be changed more high degree. Therefore, the identified model sufficiently approximates the longitudinal force of the wheel even when the LUT refers to the interpolated load.

\section{Simulation}

In this section, to evaluate the accuracy of the identified model for the rover, we design the controller system using the identified model, and confirm the response through the numerical simulation.

\subsection{Controller design}

To verify whether the rover model with terramechanics wheel model could be controlled using the identified model through the numerical simulation, we construct the

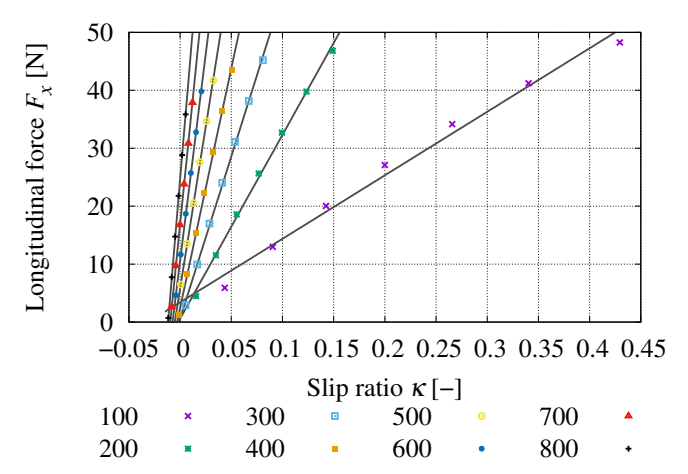

Figure 5. Reference results of the terramechanics model of slip ratio and longitudinal force obtained by step input of wheel torque and linear approximation of them.

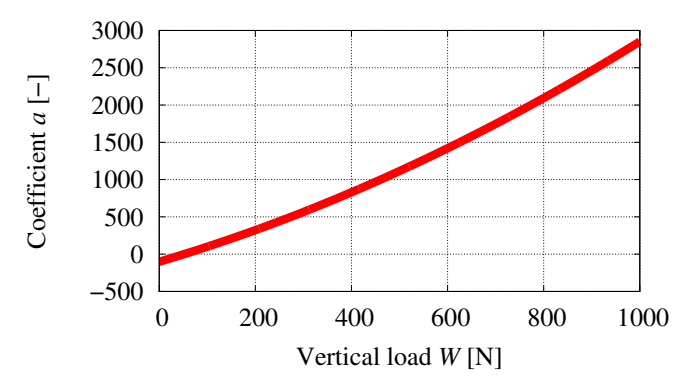

Figure 6. Coefficient $a$ of polynominal.

controller system: the identified model in section 3 is used as the control model to calculate a wheel torque from a velocity controller. Then, the torques are imposed on the rover model (plant model in section2). The system calculates the wheel torque by feedback control so that the rover achieves the target velocity. In vehicle motion controller, we regard the rover as a mass point model for calculating the rover force on the CoG. To achieve the designed motion, it is assumed that each wheel generate the same longitudinal force as follows:

$$
F_{x, \text { all }} / 6=\tilde{f}_{\mathrm{w}, i},
$$

where $F_{x, \text { all }}$ is whole longitudinal force of the rover, $\tilde{f}_{\mathrm{w}, i}$ is longitudinal force of each wheel and subscript $i=1 \sim 6$ indicates the limbs number. Each wheel torque $T_{\mathrm{w}, \mathrm{i}}$ is calculated using the inverse identified longitudinal force model. Then, to realize the inverse model which is the linear first order system, we add the second order filter in front of it so that the model become the strictly proper 


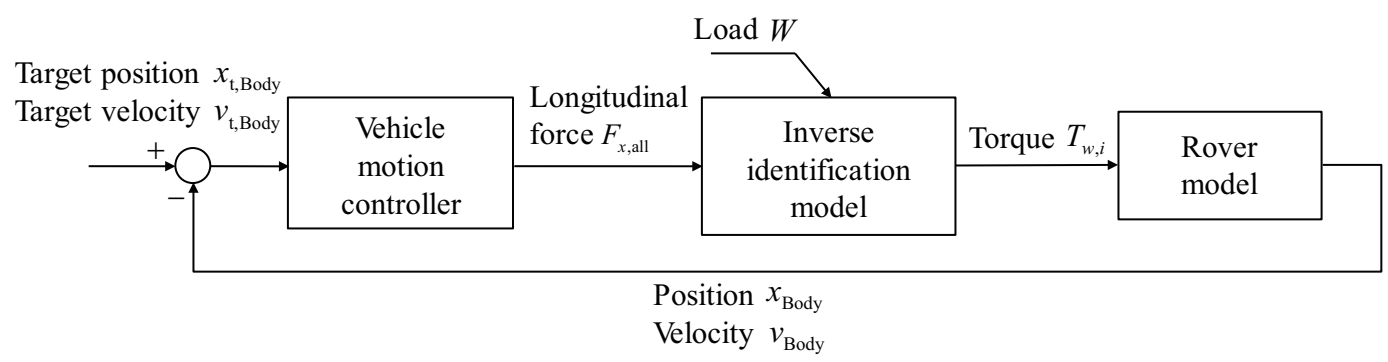

Figure 9. Controller system.

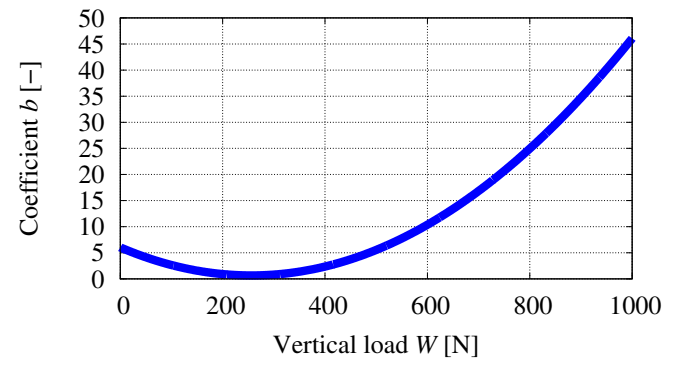

Figure 7. Coefficient $b$ of polynominal.

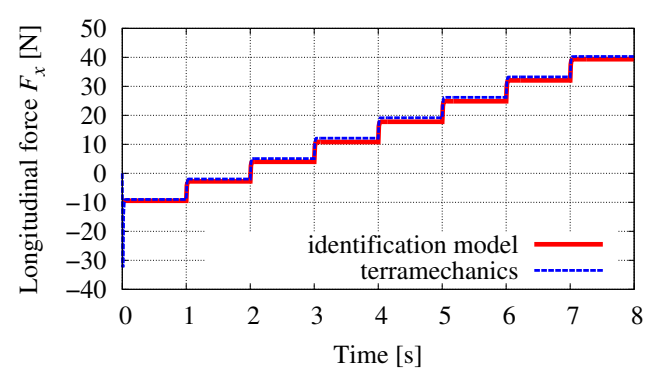

Figure 8. Example of comparisom foward identified model with terramechanics wheel model $(W=575 \mathrm{~N})$.

model. Using the inverse model, we verify the simple characteristics of the identified model when the model is applyed to the rover model. The filter is defined as follows:

$$
\frac{\omega_{\mathrm{n}}^{2}}{s^{2}+2 \omega_{\mathrm{n}} s+\omega_{\mathrm{n}}^{2}},
$$

where $\omega_{\mathrm{n}}$ is natural angular frequency and set to be $342 \mathrm{rad} / \mathrm{s}$. The calculated wheel torque is imposed on each wheel of the rover model which indicates the right block depicted in Figure 9.

\subsection{Simulation conditions}

To verify the response of the wheel model, we conduct a simulation that the rover tracks the target velocity on a plane while keeping the initial posture of the rover. The reference path is the straight line including an accelerattion areas. In this simulation, we assume that the lunar surface is covered with regolith uniformly. The parameter of rover mass, target value, soil and wheel shape are indicated in Table 2 (Ishigami et al., 2007).
Table 2. Parameter of rover, wheel and soil.

\begin{tabular}{lll}
\hline Parameter & Value & Unit \\
\hline Rover mass $M$ & 1570 & $\mathrm{~kg}$ \\
Target position $x_{\mathrm{t}, \text { Body }}$ & $1.0 \times$ time & $\mathrm{m}$ \\
Target velocity $v_{\mathrm{t}, \text { Body }}$ & 1.0 & $\mathrm{~m} / \mathrm{s}$ \\
Wheel radius $r$ & 0.355 & $\mathrm{~m}$ \\
Wheel tread $b$ & 0.175 & $\mathrm{~m}$ \\
Cohension stress $c$ & 0.80 & $\mathrm{kPa}$ \\
$a_{0}$ & 0.4 & - \\
$a_{1}$ & 0.15 & - \\
Pressure-sinkage module $k_{\mathrm{c}}$ & $1.37 \times 10^{3}$ & $\mathrm{~N} / \mathrm{m}^{n+1}$ \\
Pressure-sinkage module $k_{\phi}$ & $8.14 \times 10^{5}$ & $\mathrm{~N} / \mathrm{m}^{n+2}$ \\
Soil deformation module $k_{x}$ & 0.036 & $\mathrm{~m}$ \\
Sinkage exponent $n$ & 1.0 & - \\
Friction angle $\phi$ & 37.2 & $\mathrm{deg}$ \\
Wheel sinkage ratio $\lambda$ & 0.90 & - \\
\hline
\end{tabular}

\subsection{Results and discussions}

The simulation results using the identified model are shown in Figure 10. Since the rover moves on a straight line and arranges a symmetric leg position in this simulation condition, we plot the results of the Limb1 $\sim 3$. Figure 10 (a) through (h) depict the wheel torque, the wheel resistance torque, the vertical force of each wheel, each wheel sinkage, the slip ratio of each wheel, the longitudinal force of each wheel, the velocity of the rover and the desired longitudinal force, respectively.

As shown in Figure 10 (a), the identified model calculates the wheel torque considering the influence of resistance torque, so that the rover enable the wheel to drive smoothly. It is because the controller implicitly considers the effect of resistance which is depicted in Figure 10 (b).

The inertia force due to the acceleration influences that the load distribution of the wheel biases backward of the rover. As a result, Figure 10 (c) indicates that, during the acceleration, the vertical force of the Limb 3 increases while that of the Limb 1 decreases. The load change affects the change of the wheel sinkage $h$ as depicted in Figure $10(\mathrm{~d})$. The wheel sinkage $h$ is adapted to the vertical force, so that the physical adequacy of the wheel model based on terramechanics can be confirmed. The wheel torque is calculated using the identified model, so that 
Table 3. RMS error of longitudinal force.

\begin{tabular}{llll}
\hline & Limb 1 & Limb 2 & Limb 3 \\
\hline RMS error & $0.7 \mathrm{~N}$ & $0.4 \mathrm{~N}$ & $0.5 \mathrm{~N}$ \\
\hline
\end{tabular}

the wheel arises the different slip ratio, as shown in Figure 10 (e). Accordingly, the longitudinal force of the each wheel is generated uniformly as depicted in Figure 10 (f) even when the load of the each wheel is different. Figure $10(\mathrm{~g})$ indicates that the rover accelerates until the translational velocity reaches $1.0 \mathrm{~m} / \mathrm{s}$. Figure $10(\mathrm{~h}) \mathrm{de}-$ picts the actual and desired longitudinal force of limb 1 as a representative example. Table 3 shows the RMS error between the actual and desired longitudinal force in $\operatorname{limb} 1 \sim 3$. This difference in the longitudinal force is caused by the approximation error of the identified model. The precision of the identified model tends to lower as the load decreases, as shown in Table 1. Thus, since the load of Limb 1 decreases during the acceleration, the RMS error becomes the largest. The maximum longitudinal force reaches about $30 \mathrm{~N}$. Nevertheless, all RMS error is below $1.0 \mathrm{~N}$. Although the actual longitudinal force is not equal to the desired, the influence is adequately suppressed by the feedback control.

Each wheel can generate the desired longitudinal force due to calculating the wheel torque corresponding to load change. Moreover, through the use of the identified model, the wheel torque considering the influence of loose soil can be obtained without the optimal calculation for a decision of the wheel sinkage. Therefore, it is shown that the identified longitudinal force model based on the more detailed model has the high accuracy when the model is applied to the rover controller.

\section{Conclusions}

In this paper, we construct the wheel model based on terramechanics derived from semi-empirical model by using Modelica language. In order to consider the longitudinal force of the constructed wheel model, we approximate it by the linear first order system. Designing the controller using the identified model, we investigate the influence on driving systems of the rover moving on loose soil. The simulation results indicate that the identified model can adapt the influence of load change and consider the soil deformation, so that the identified model has a high accuracy. With reference to the model used in the control, it is important to simplify the structure and identify the characteristic. Consequently, the use of the identified longitudinal force model contributes to a control design for the rover.

As for the problems to be solved from now on, to enhance the mobility on loose soil, the lateral force of the wheel should be identified to design a controller.

\section{Acknowledgments}

The authors gratefully acknowledge the support of Grant in Aid for Scientific Research (C) No.15K06155 of Japan.

\section{References}

Liang Ding, Hai-bo Gao, Zong-quan Deng, Zhijun Li, Ke-rui Xia, and Guang-ren Duan. Path-following control of wheeled planetary exploration robots moving on deformable rough terrain. The Scientific World Journal, 2014, 2014.

Kanfeng Gu, Yingzi Wei, Hongguang Wang, and Mingyang Zhao. Dynamic modeling and sliding mode driving control for lunar rover slip. In Integration Technology, 2007. ICIT'07. IEEE International Conference on, pages 36-41. IEEE, 2007.

Karl Iagnemma and Steven Dubowsky. Traction control of wheeled robotic vehicles in rough terrain with application to planetary rovers. The international Journal of robotics research, 23(10-11):1029-1040, 2004.

Genya Ishigami, Akiko Miwa, Keiji Nagatani, and Kazuya Yoshida. Terramechanics-based model for steering maneuver of planetary exploration rovers on loose soil. Journal of Field robotics, 24(3):233-250, 2007.

Rainer Krenn and Andreas Gibbesch. Soft soil contact modeling technique for multi-body system simulation. In Trends in computational contact mechanics, pages 135-155. Springer, 2011.

H Nakashima, H Fujii, A Oida, M Momozu, H Kanamori, S Aoki, T Yokoyama, H Shimizu, J Miyasaka, and K Ohdoi. Discrete element method analysis of single wheel performance for a small lunar rover on sloped terrain. Journal of Terramechanics, 47(5):307-321, 2010.

Tiziano Pulecchi and Marco Lovera. A modelica library for space flight dynamics. In In Proceedings of the 5th International Modelica Conference. Citeseer, 2006.

Aravind Seeni, Bernd Schafer, Bernhard Rebele, and Nikolai Tolyarenko. Robot mobility concepts for extraterrestrial surface exploration. In Aerospace Conference, 2008 IEEE, pages 1-14. IEEE, 2008.

Sh Taheri, C Sandu, S Taheri, E Pinto, and D Gorsich. A technical survey on terramechanics models for tire-terrain interaction used in modeling and simulation of wheeled vehicles. Journal of Terramechanics, 57:1-22, 2015.

Brian H. Wilcox, Todd Litwin, Jeff Biesiadecki, Jaret Matthews, Matt Heverly, Jack Morrison, Julie Townsend, Norman Ahmad, Allen Sirota, and Brian Cooper. ATHLETE: A cargo handling and manipulation robot for the moon. Journal of Field Robotics, 27(5):421-434, 2007.

Jo Yung Wong. Theory of ground vehicles. John Wiley \& Sons, 2001.

Hiroki Yoshikawa, Takatsugu Oda, Kenichiro Nonaka, and Kazuma Sekiguchi. Modeling and simulation for leg-wheel mobile robots using modelica. In The First Japanese Modelica Conferences, May 23-24, Tokyo, Japan, number 124, pages 55-60. Linköping University Electronic Press, 2016. 


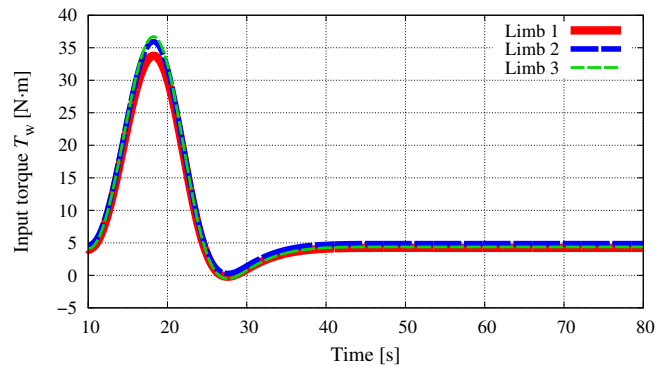

(a) Wheel torque of each wheel.

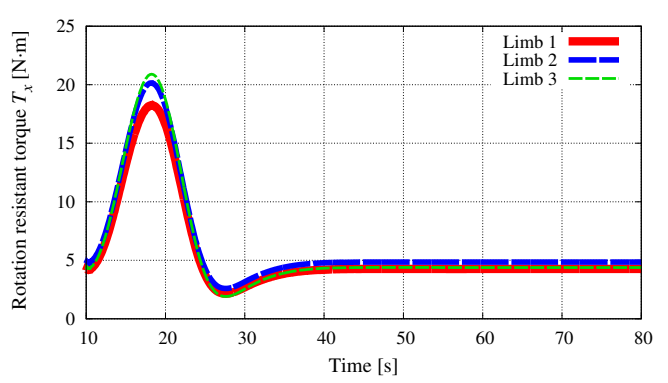

(b) Rolling resistance torque of each wheel.

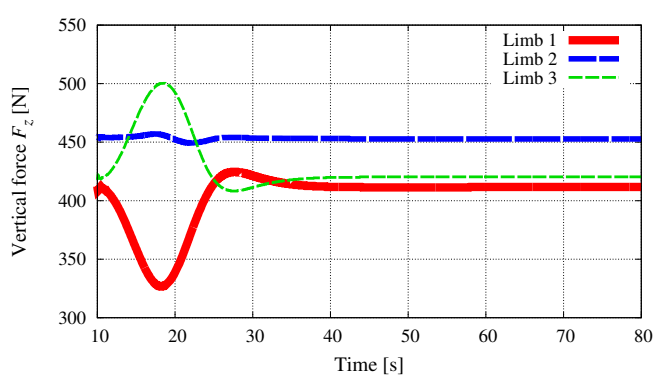

(c) Vertical force of each wheel.

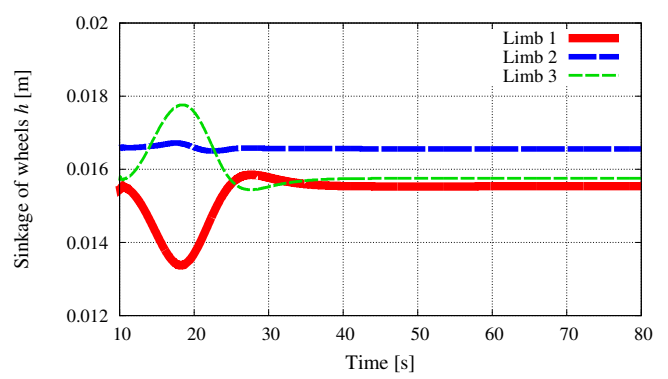

(d) Sinkage of each wheel.

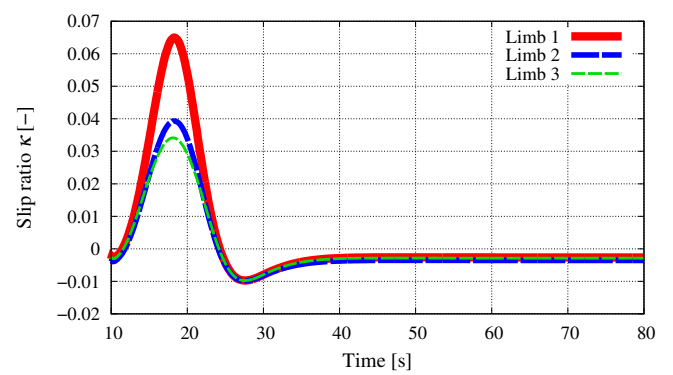

(e) Slip ratio of each wheel.

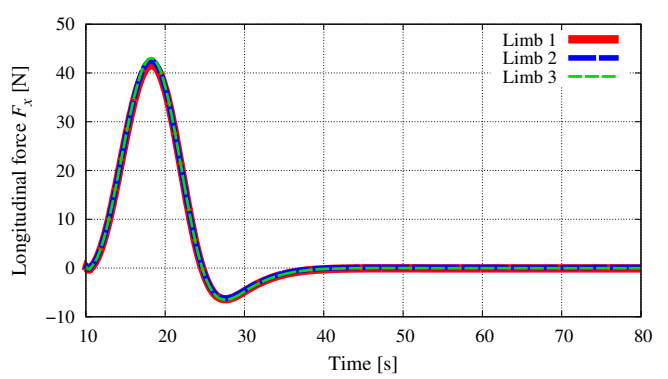

(f) Longitudinal force of each wheel.

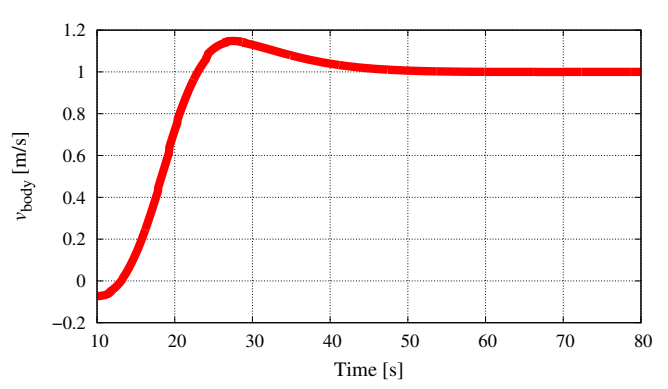

(g) Velocity of rover model.

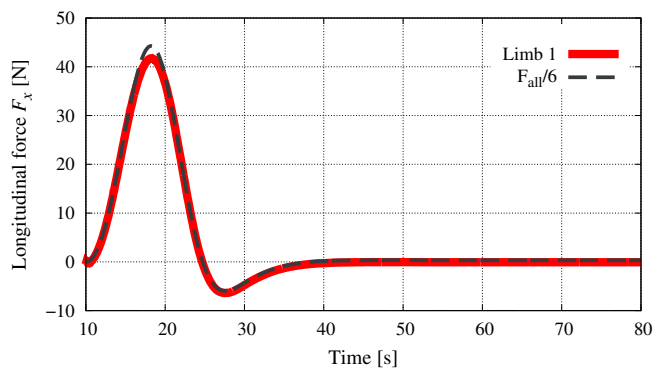

(h) Actual and desired longitudinal force of limb 1.

Figure 10. Rover driving simulation using longitudinal force model considring terramechanics for driving force distribution. 